\title{
COMMUNITY STRUCTURE IN JAZZ
}

\author{
PABLO M. GLEISER ${ }^{1}$ and LEON DANON ${ }^{2}$ \\ ${ }^{1}$ Departament de Física Fonamental, \\ Universitat de Barcelona, Diagonal 647, \\ 08028 Barcelona, Spain. gleiser@ffn.ub.es. \\ ${ }^{2}$ Departament de Física Fonamental, \\ Universitat de Barcelona, Diagonal 647, \\ 08028 Barcelona, Spain. ldanon@ffn.ub.es.
}

\begin{abstract}
Using a database of jazz recordings we study the collaboration network of jazz musicians. We define the network at two different levels. First we study the collaboration network between individuals, where two musicians are connected if they have played in the same band. Then we consider the collaboration between bands, where two bands are connected if they have a musician in common. The community structure analysis reveals that these constructions capture essential ingredients of the social interactions between jazz musicians. We observe correlations between recording locations, racial segregation and the community structure. A quantitative analysis of the community size distribution reveals a surprising similarity with an e-mail based social network recently studied.
\end{abstract}

PACS numbers: 89.75.Fb 89.75.Da 89.75.Hc 


\section{INTRODUCTION}

In the last years the physics community has devoted a strong effort to the study of social networks 1]. The availability of large databases containing information on the collaborations between movie actors, scientists, etc. has allowed for many statistical properties of these networks to be characterized. [2]. They revealed that some characteristics appear to be general for these kind of networks. In particular they show the so called small world property, that is the average distance between vertices is small, while the clustering of vertices remains high.It has also been observed that the degree distribution $P(k)$ is skewed. In the particular case where $P(k)$ presents a power law tail the network is known as scale free. [3].

An interesting point which has recently been brought to attention is the community structure of networks. Communities appear in networks where vertices join together in tight groups that have few connections between them. By eliminating these connections it is possible to isolate the communities. In fact this is the main idea behind the algorithm that Girvan and Newman have recently proposed [4].

In this work we study the topology and the community structure of the collaboration network of jazz musicians. In order to do so we construct the network at two different levels. First we build the network from a 'microscopic' point of view. In this case each vertex corresponds to a musician, and two musicians are connected if the have recorded in the same band. Then we build the network from a 'coarse-grained' point of view. In this case each vertex corresponds to a band, and a link between two bands is established if they have at least one musician in common. Clearly, this is the most simple way in which one can establish a connection between bands, and the definition can be extended to incorporate directed and/or weighted links. However, we show that even by using this simple definition we are able to recover essential ingredients of the social interactions between the musicians.

We characterize the topology of these networks by studying the degree distributions, clustering and average nearest neighbors degree. A comparison of the quantitative properties of the musicians and bands networks reveals differences which are analyzed in detail. We also study the community structure of both networks. This analysis reveals the presence of communities which have a strong correlation with the recording locations of the bands, and also shows the presence of racial segregation between the musicians. These characteristics are well documented in jazz history [5]. 
Finally, we characterize quantitatively the community size distribution $P(s)$. The shape of this distribution presents a surprising similarity with the results reported in a recent study of the e-mail network in the University Rovira i Virgili in Tarragona, Spain [6].

\section{THE JAZZ NETWORK}

The data was obtained from The Red Hot Jazz Archive digital database 7]. In our analysis we include 198 bands that performed between 1912 and 1940, with most of the bands in the 1920's. The database lists the musicians that played in each band without distinguishing which musicians played at different times, so it is not possible to study the time evolution of the collaboration network. The bands contain 1275 different names of musicians. However, it is important to stress that this number does not necessarily represent the number of individuals, similar to the comment noted in [8]. The same musician might appear with different names in the database. For example, the musician Henry Allen appears as Henry Allen, Red Allen or Henry Red Allen. Also in some bands the names of some musicians are not known. As a consequence they are cited in the database with the same name: unknown. In Fig. 1 we show the distribution of musicians that have played in a band. It presents a skewed shape, with a peak around 5-10 musicians and a few large bands that include up to 171 musicians.

Let us begin with the collaboration network of individuals. Since some musicians have played in more than one band we obtain a modular network, formed by fully connected modules (bands) which are also connected between each other. Using this definition we obtain a network with a mean distance of 2.79 and, as we show later, with very high clustering. The bands network is also a small world, with a mean distance of 2.26 , slightly smaller than the distance between musicians. This is an expected result, since in the musicians network an individual may need to contact a musician in his band in order to reach a musician in another band.

We measure the typical quantities used to characterize complex networks. First we study the degree distribution and its correlations. Then we look at the clustering coefficient and how it depends on the degree $k$. In Fig. 2 we show the cumulative degree distribution $P(k)$, i.e. the probability for a vertex to have a degree larger or equal to $k$. In the musicians network, shown in Fig. 2(a), a slow decay with a power law tail $P(k) \sim\left(1+a_{0} k\right)^{-\alpha_{m}}$ 
with $a_{0}=0.022(1)$ and $\alpha_{m}=1.38(1)$ is observed for values of $k$ up to $k=170$. This value coincides with the degree of the musicians which have only played in Paul Whiteman's Orchestra, the band that includes the largest number of musicians. For $k>170$ a faster decay is observed. In table I we present the names of the ten most connected musicians with their corresponding $k$ values.

Fig. 2(a) reveals that there are few musicians which have a very large number of connections. Does this mean that these musicians play the role of hubs, and connect a large number of bands or is it simply that they have just played in the few bands which include the largest number of musicians? One way to tackle this question is to eliminate all the links between musicians in the same band and consider only the links between bands. This is precisely what we do when we build the bands network. In Fig. 2(b) we present the behavior of $P(k)$ for the bands network. A clear stretched exponential behavior $P(k) \sim \exp \left(-\left(k / k^{*}\right)^{\alpha_{b}}\right)$ with $k^{*}=32.8(1)$ and $\alpha_{b}=1.78(1)$ is observed. This result suggests that the bands are interconnected with a characteristic number of links. However we should keep in mind the fact that in the bands network construction two bands are connected by just one link, although

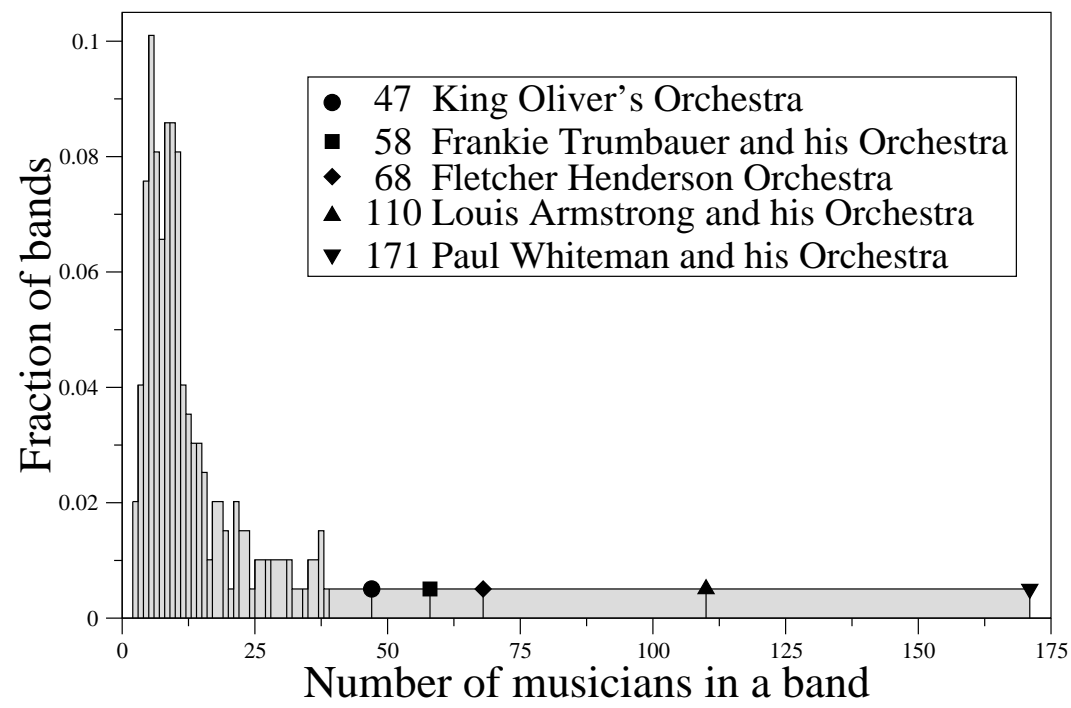

FIG. 1: Distribution of musicians per band. The horizontal axis corresponds to the number of musicians that have played in a given band, while the vertical axis presents the fraction of bands with the given number of musicians. The inset shows the names of the five bands that include the largest number of musicians. 


\begin{tabular}{ccc}
\hline \hline Position & Name & $\mathrm{k}$ \\
\hline 1 & Eddie Lang & 415 \\
2 & Jack Teagarden & 387 \\
3 & Frank Signorelli & 332 \\
4 & Frankie Trumbauer & 307 \\
5 & Joe Venuti & 295 \\
6 & Bob Mayhew & 287 \\
7 & Bill Rank & 267 \\
8 & Fud Livingston & 265 \\
9 & Miff Mole & 262 \\
10 & Louis Armstrong & 262
\end{tabular}

TABLE I: The ten most connected musicians with the corresponding degree

they may share more than one musician. Clearly, just by analyzing the degree distributions we cannot answer the question. In order to advance one step forward we will study the correlations present in the networks.
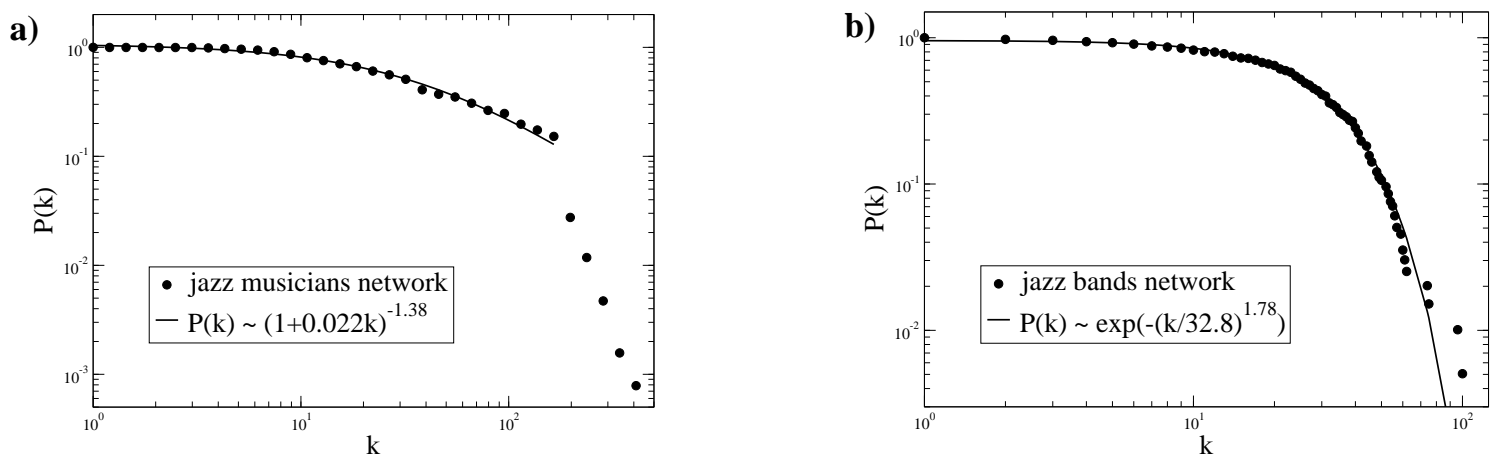

FIG. 2: Cumulative degree distribution $P(k)$ for the jazz musicians network (a) and the jazz bands network (b).

It has been observed that social networks show "assortative mixing" on their degrees, that is, there is a preference for high degree vertices to connect to other high degree vertices 9]. In order to characterize the degree correlations present in the jazz collaboration network we study the behavior of the average nearest neighbors degree of the vertices of degree $k$ 
a)

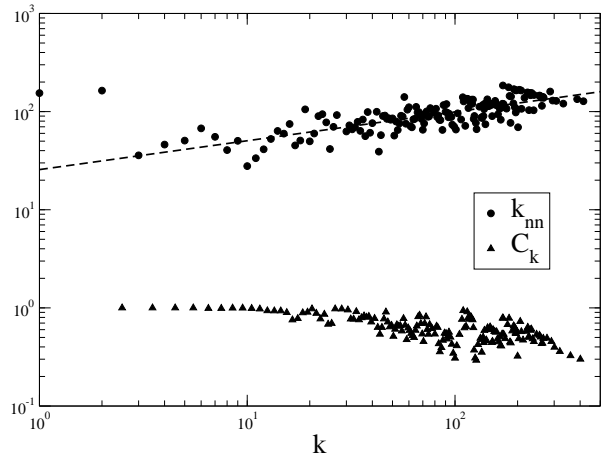

b)

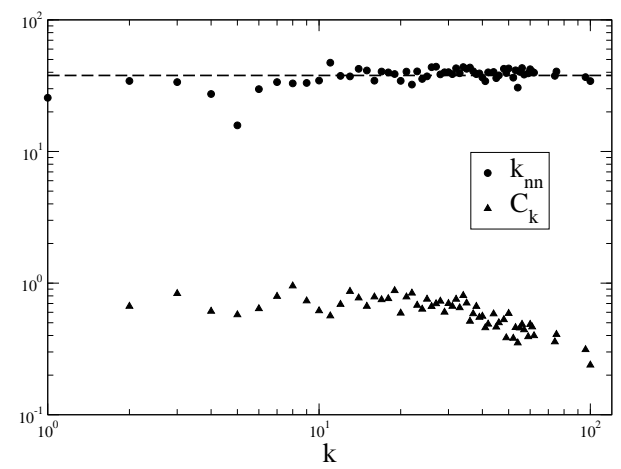

FIG. 3: Average degree $k_{n n}$ of nearest neighbors of vertices with degree $k$ (full circles) and clustering coefficient $C_{k}$ (full triangles) vs. $k$ for the musicians network (a), and the bands network (b). The dashed lines are guides to the eye.

10], defined as

$$
k_{n n}(k) \equiv \sum_{k^{\prime}} k^{\prime} P\left(k^{\prime} \mid k\right)
$$

where $P\left(k^{\prime} \mid k\right)$ is the conditional probability that a vertex of degree $k$ is connected to a vertex of degree $k^{\prime}$. Fig. 3(a) shows the behavior of $k_{n n}$ as a function of $k$ for the musicians network. A clear increase in $k_{n n}$ is observed as $k$ grows. For $k<170$ this could simply reflect the trivial fact that musicians are already grouped in bands of different sizes. We known that the musicians with $k>170$ have more links than the ones they get simply by playing in one band. However, when we consider only these musicians the behavior of $k_{n n}$ is very noisy, and no tendency can be extracted. Fig. 33(b) shows the behavior of $k_{n n}$ for the bands network. In this case no correlation is observed, and $k_{n n}$ fluctuates around a constant value independent of $k$. Again we observe that both networks present different results which do not help us to understand the role played by the most connected musicians.

We have also measured the clustering coefficient $C_{k}$ around nodes of degree $k$, which gives the fraction of neighbors of nodes with degree $k$ that are linked. Fig. 3)(a) shows that the musicians network is highly clustered. $C_{k} \simeq 1$ up to $k \approx 30$, and then presents a slow decay with oscillations. The peaks of these oscillations coincide with the $k$ values of the large bands. This result is expected by construction, since the musicians network is formed by fully connected modules which have $C=1$ by definition. The bands network also presents a large clustering with $C_{k} \simeq 1$ up to $k \approx 30$ followed by a slow decay.

In this section we have analyzed the typical quantities which are used to characterize the 
statistical properties of networks. The analysis and comparison of the results obtained leaves a number of open questions. On one hand the degree distribution of the musicians network shows a skewed shape, with a power law tail and a small number of musicians with a very large number of connections. On the other hand the bands network presents a stretched exponential distribution revealing that they are interconnected with a characteristic number of links. The correlations also show different properties, on one hand the musicians network shows a positive correlation which seems to indicate assortative mixing in the degrees, while the bands network does not present any correlations at all.

How can we be confident that we are truly studying the collaboration network of jazz musicians? Are the different ways of constructing the networks creating misleading artificial artifacts or do they give us a real insight into the collaboration structure? In the next section we use the community structure analysis to answer these questions. As we show the different constructions do capture essential ingredients of the collaborations between the musicians. Also the community structure analysis allows for a quantitative characterization of the network other than the ones presented in this section.

\section{COMMUNITY STRUCTURE OF THE JAZZ NETWORK}

To analyze the community structure we use the method proposed by Girvan and Newman

4]. The algorithm is based on the concept of edge betweenness [11], which is defined as the number of minimum paths connecting pairs of nodes that go through that edge. The main idea is that edges which connect highly clustered communities have a higher edge betweenness, and therefore cutting these edges should separate communities. The algorithm proceeds as follows:

1. The edge betweenness of every edge in the network is calculated

2. The edge with the highest betweenness is removed. If more than one edge has the highest value then one of them is chosen at random.

3. All the betweennesses are recalculated and step 2 is repeated until no edges remain.

In order to describe the splitting process we generate a binary tree, as described in [6], where bifurcations depict communities and leaves represent the actual vertices of the 


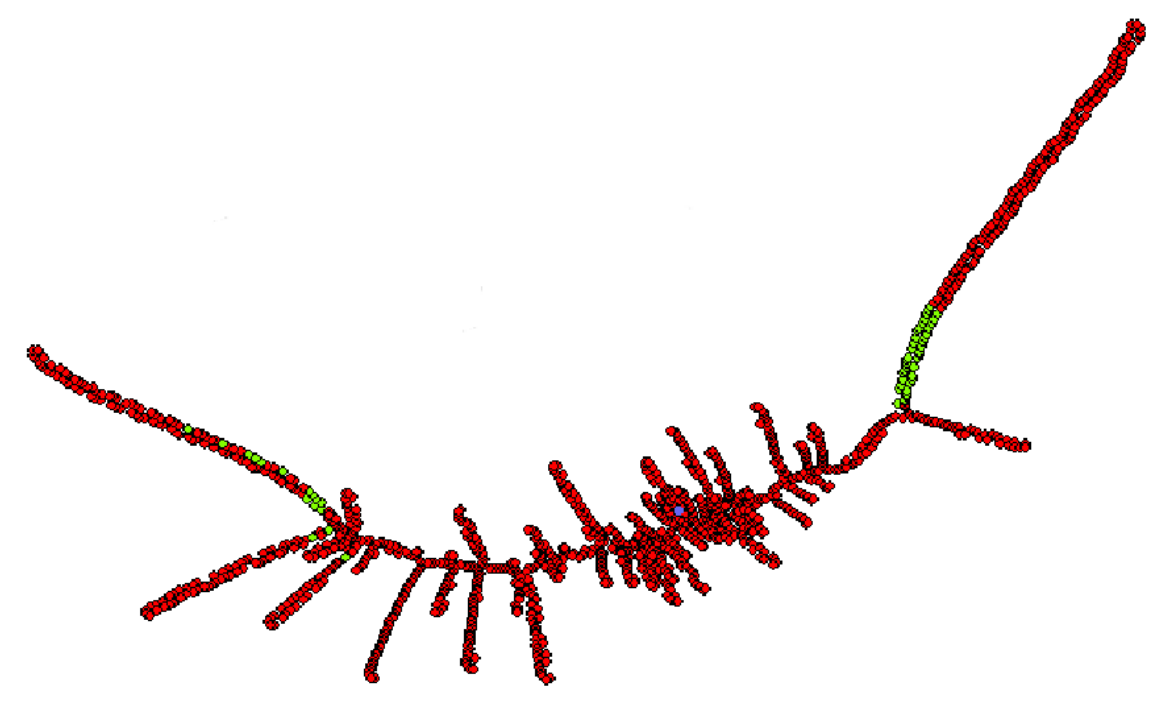

FIG. 4: Community structure of the jazz musicians network. The root of the tree, in the middle of the figure, is indicated with the color blue. The musicians with $k>170$ are indicated with green.

network. Note however, that since the musicians network is constructed by connecting fully connected modules, the tips of the branches do not necessarily correspond to the most central musicians in the network. In fact, once a band is disconnected from the large component all its edges are completely equivalent.

In Fig. 4 we show the binary tree corresponding to the musicians network. The root of the tree is indicated with a blue circle. Going from the root of the tree to its tip, we note that first a few small branches separate. A little further on, the tree splits into two large branches or communities, which then split further into smaller ones. This separation into two distinct communities can be interpreted as the manifestation of racial segregation which was present at that time. Although a small number of collaborations existed between races, most bands were exclusively comprised of one race or the other. As a consequence a division in two large communities separating black and white musicians should be present. In fact, an analysis of the names of the musicians shows that the musicians on the left community are black while the musicians on the right are white.

In the previous section we have shown that the musicians network presents a positive correlation on the degrees. However, it was not clear if this result indicated the presence of assortative mixing on the degree or simply reflected the way in which the network was constructed. Let us analyze this point using the community structure results. In Fig. 4 
we indicate with color green the musicians with $k>170$. A visual inspection of the figure reveals that these musicians are divided mainly into two communities. An analysis of the names of these 55 musicians [12] reveals that the musicians in the three communities on the left of the figure are only black musicians, while the musicians in the right community are all white. This results shows that the network presents assortative mixing on the degree, and also shows a clear racial segregation at least for the most connected musicians.

Let us analyze now the community structure of the bands network. In Fig. 5] we show the binary tree obtained using the Girvan Newman algorithm. The bands network reveals a very simple community structure. The tree is roughly divided into two large communities as expected. However, the largest community also splits into two communities. In order to show the origin of this division we have analyzed the cities where the bands recorded. In Fig. 5 we indicate with color red the bands that have recorded in New York. The bands that recorded in Chicago are indicated with blue. Clearly Chicago and New York dominate as preferred recording locations. The figure shows that the recording locations play a fundamental role in the division of communities. Note that New York appears in two different branches as a preferred recording location. A closer look at the names of the bands in the different communities reveals again a racial segregation between black and white bands. The upper branch correspond to bands with white musicians, while the lower branches correspond to bands with black musicians.

Let us resume the results we obtained in this section. Fig. 4 clearly shows that the musicians network is assortative on the degree. It also reflects racial segregation between musicians. We have also seen that the division into communities presents a strong correlation with the geographical locations where the bands recorded. These results show that the musicians and bands network capture essential ingredients of the collaboration network of jazz musicians. With this in mind we characterize quantitatively the community structure. In order to do so we calculate the cumulative community size distribution $P(s)$ as in [6]. This quantity gives the probability of a community to have a size larger or equal to $s$. Fig. [6] shows that this distribution is heavily skewed, with a slow decay for community sizes up to $s \sim 200$, which roughly corresponds to the size of the largest band. This is followed by a faster decay and a cutoff corresponding to the size of the system at $s \sim 1000$. A comparison of the shape of $P(s)$ with the results obtained in a recent study of the e-mail network of University Rovira i Virgili [6] shows a surprising similarity. In fact, as shown in Fig. 6, both 


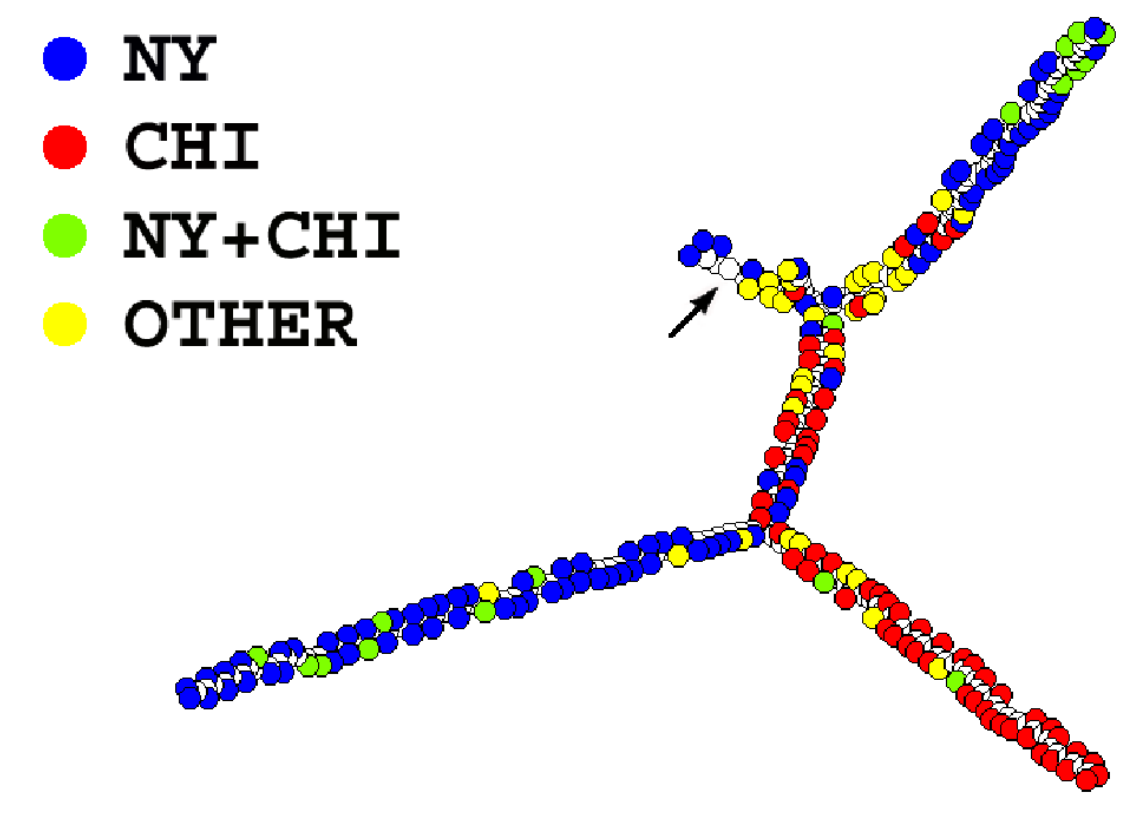

FIG. 5: Communities in the jazz bands network. The arrow indicates the root of the tree. The different colors correspond to cities where a band has recorded: New York (blue), Chicago (red), both in New York and Chicago (green) and other cities (yellow).

networks present a power law decay with the same exponent -0.48 up to $s \sim 200$. For small values of $s$ the jazz network deviates from this behavior, reflecting the fact that musicians are already grouped in bands of a certain size (see Fig 11), an effect which is not present in the e-mail network.

This result leaves a number of open questions. Are there fundamental laws regarding the social interactions that lead to the formation of community structures? Can these structures be characterized quantitatively? We hope that future work along these lines will help us to answer these interesting questions.

\section{Acknowledgments}

The authors acknowledge financial support from the Spanish Ministerio de Educación, Cultura y Deporte, DGES (Grant No. BFM2000-0626) and European Commission - Fet Open project COSIN IST-2001-33555. They wish to thank Fede Bartumeus, Marián Boguná, Albert Díaz Guilera, Conrad Pérez and Enric Vázquez for useful comments and suggestions, as well as Roger Guimerà and Alex Arenas for the original code. P. M. G. thanks Fundación 


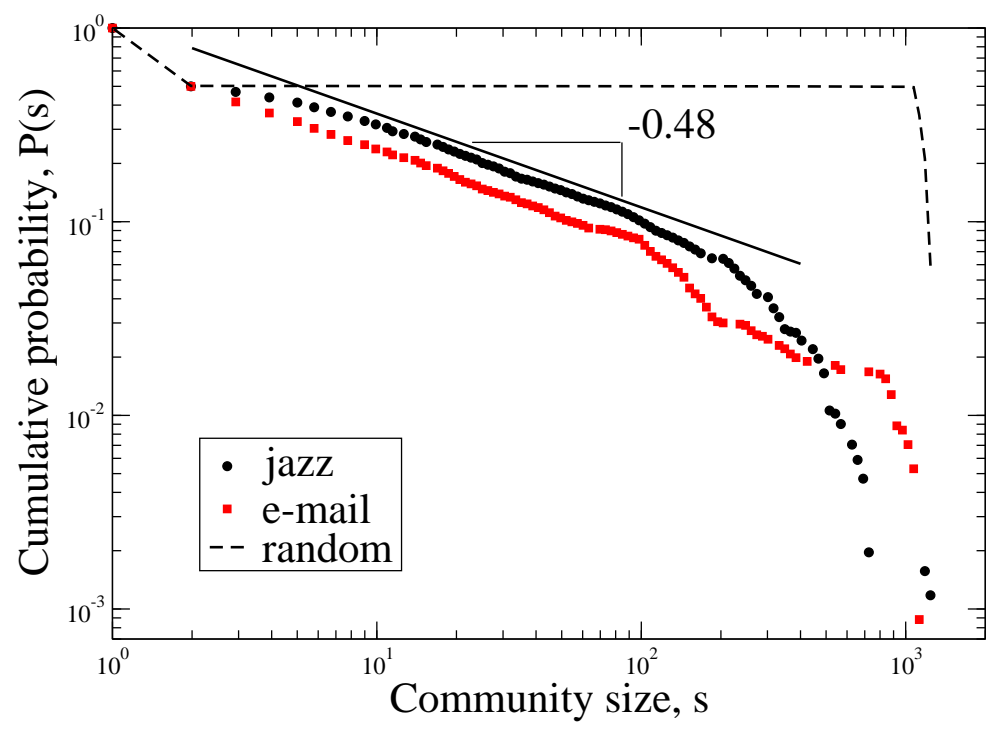

FIG. 6: Community size distribution $P(s)$ as a function of community size $s$. The results for the jazz musicians network are plotted in full circles, while full triangles correspond to the e-mail network of University Rovira i Virgili. The dotted line corresponds to the results obtained in a random network with the same degree distribution as the musicians network.

Antorchas and L. D. thanks Generalitat de Catalunya for financial support.

[1] M.E.J. Newman, The structure and function of complex networks. SIAM Review 45, 167-256 (2003).

[2] Reka Albert, Albert-Laszlo Barabasi, Statistical mechanics of complex networks. Reviews of Modern Physics 74, 47 (2002).

[3] Reka Albert, Hawoong Jeong, and Albert-Laszle Barabasi Diameter of the World Wide Web, Nature 401, 130-131 (1999).

[4] M. Girvan and M.E.J. Newman, Community structure in social and biological networks. Proc. Natl. Acad. Sci. USA 99 7821-7826 (2002).

[5] The New Grove Dictionary of Jazz (St. Martin's Press, New York, 1994).

[6] R. Guimerà, L. Danon, A. Díaz-Guilera, F. Giralt and A. Arenas, Self-similar community structure in organizations. arXiv:cond-mat/0211498

[7] The Red Hot Jazz Archive, available at http://www.redhotjazz.com 
[8] M. E. J. Newman, Who is the best connected scientist? A study of scientific coauthorship networks. Phys.Rev. E 64 (2001) 016131; Phys.Rev. E 64 (2001) 016132.

[9] M.E.J. Newman, Assortative mixing in networks. Phys. Rev. Lett. 89, 208701 (2002).

[10] Romualdo Pastor-Satorras, Alexei Vázquez, and Alessandro Vespignani, Dynamical and Correlation Properties of the Internet. Phys. Rev. Lett. 87, 258701 (2001).

[11] L. Freeman, A set of measures of centrality based upon betweenness, Sociometry, 40 35-41 (1977).

[12] For a complete list of the names of the musicians with their connectivities see http://www.ffn.ub.es/ gleiser 